\title{
The Role of Molecular Dications in Planetary Atmospheric Escape
}

\author{
Stefano Falcinelli 1,*, Fernando Pirani ${ }^{2}$, Michele Alagia ${ }^{3}$, Luca Schio ${ }^{3}$, Robert Richter ${ }^{4}$, \\ Stefano Stranges ${ }^{3,5}$, Nadia Balucani ${ }^{2}$, and Franco Vecchiocattivi ${ }^{1}$ \\ Published: 16 July 2016 \\ 1 Department of Civil and Environmental Engineering, University of Perugia, Via G. Duranti 93, \\ 06125 Perugia, Italy; franco@vecchio.it \\ 2 Department of Chemistry, Biology and Biotechnologies, University of Perugia, Via Elce di Sotto, \\ 8, 06123 Perugia, Italy; fernando.pirani@unipg.it (F.P.) \\ 3 IOM-CNR Tasc, Km 163.5, Area Science Park, 34149 Basovizza, Trieste, Italy; \\ michele.alagia@elettra.eu (M.A.); luca.schio90@gmail.com (L.S.); stefano.stranges@uniroma1.it (S.S.) \\ 4 Elettra-Sincrotrone Trieste, Area Science Park, 34149 Basovizza, Trieste, Italy; robert.richter@elettra.eu \\ 5 Department of Chemistry and Drug Technology, University of Rome "La Sapienza", 00185 Rome, Italy \\ * Correspondence: stefano.falcinelli@unipg.it; Tel.: +39-75-585-3856
}

\begin{abstract}
Fundamental properties of multiply charged molecular ions, such as energetics, structure, stability, lifetime and fragmentation dynamics, are relevant to understand and to model the behavior of gaseous plasmas as well as ionosphere and astrophysical environments. Experimental determinations of the Kinetic Energy Released (KER) for ions originating from dissociations reactions, induced by Coulomb explosion of doubly charged molecular ions (molecular dications) produced by double photoionization of $\mathrm{CO}_{2}, \mathrm{~N}_{2} \mathrm{O}$ and $\mathrm{C}_{2} \mathrm{H}_{2}$ molecules of interest in planetary atmospheres, are reported. The KER measurement as a function of the ultraviolet (UV) photon energy in the range of $28-65 \mathrm{eV}$ are extracted from the electron-ion-ion coincidence spectra obtained by using tunable synchrotron radiation coupled with ion imaging techniques at the ELETTRA Synchrotron Light Laboratory Trieste, Italy. These experiments allow assessing the probability of escape for simple ionic species in the upper atmosphere of Mars, Venus and Titan. The measured KER in the case of $\mathrm{H}^{+}, \mathrm{C}^{+}, \mathrm{CH}^{+}, \mathrm{CH}_{2}+\mathrm{N}^{+}, \mathrm{O}^{+}, \mathrm{CO}^{+}, \mathrm{N}_{2}{ }^{+}$and $\mathrm{NO}^{+}$fragment ions are ranging between 1.0 and $5.5 \mathrm{eV}$, being large enough to allow these ionic species in participating in the atmospheric escape from such planets into space.
\end{abstract}

Keywords: molecular dications; astrochemistry; planetary atmospheres; ionospheres; synchrotron radiation; ions escape; mass spectrometry; coincidence technique; ion imaging

\section{Introduction}

Gas phase ionization processes induced by energetic photons, electrons or by excited metastable neutral species (also called collisional autoionization reactions [1,2]) play an important role in several phenomena occurring in low energy ionized plasmas, electric discharges $[3,4]$. Furthermore, ionic species are extremely important in the upper atmosphere of planets, where they govern the chemistry of ionospheres [5,6]. In particular, the ionosphere chemistry of Titan has recently been revealed to be extremely active by the instruments on board Cassini $[7,8]$. Finally, molecular ions have also been detected in comet tails [9].

In space, ions are formed in various ways, the importance of which depends on the specific conditions of the extraterrestrial environment considered [10-12]. The interaction of neutral 
The 1st International Electronic Conference on Atmospheric Sciences (ECAS 2016), 16-31 July 2016; Sciforum Electronic Conference Series, Vol. 1, 2016

molecules with cosmic rays, UV photons, $\mathrm{X}$-rays and other phenomena such as shock waves are all important processes for their production. In particular, the absorption of UV photons with an energy content higher than the ionization potential of the absorbing species can induce ionization with the formation of both singly and doubly charged ions. In the latter case we have the so called molecular dications. These ionic species can be produced by different techniques, as mass spectrometry [13], ion-molecule reactions [14], and double photoionization processes [15-18]. They can be stable or metastable $[13,19]$ and can be used, in principle, as energy storage at a molecular level $[13,14,20]$. Multiply charged ionic species can be produced also by cosmic rays, which are significant since they are ubiquitous and carry a large energy content (up to $100 \mathrm{GeV}$ ). They consist of protons, alpha particles, electrons, $\gamma$-rays and (to a small extent) also heavier nuclei (such as $\mathrm{C}^{6+}$ ). Furthermore, single or double ionization can occurs also by absorption of X-rays. In this case the ejection of a core electron followed by the Auger emission of another electron, produces molecular dications, which have been suggested to play a role in the envelope of young stellar objects [21] and upper planetary atmospheres [22-25].

In general, the double ionization producing a molecular dication can induce Coulomb explosion and fragment ions formation with a high kinetic energy content. For such a reason molecular dications are considered as exotic species, and when they are formed in planetary ionospheres the possibility to generate dissociative products with a kinetic energy of several $\mathrm{eV}$, allows these ionic fragments to reach sufficient velocity to escape into space. Therefore, double ionization processes can in principle contribute to the continuous erosion of the atmosphere of some planets of the Solar System, like Mars and Titan (the largest satellite of Saturn), as discussed in the following sections.

\section{Experiments}

The data here presented and discussed have been recorded by double photoionization experiments performed at the ELETTRA Synchrotron Light Laboratory (Trieste, Italy) using the ARPES (Angle-Resolved Photoemission Spectroscopy) end station of the Gas Phase Beamline. Details about the beamline and the end station have been already reported elsewhere [26], and the apparatus used for the experiment discussed here has also been described previously [27,28]. Therefore, only some features relevant for the present investigation are outlined here.

As it can be seen in Figure 1, the monochromatic energy from the selected synchrotron light beam crosses an effusive molecular beam of $\mathrm{CO}_{2}, \mathrm{~N}_{2} \mathrm{O}$ and $\mathrm{C}_{2} \mathrm{H}_{2}$ neutral precursors, and the product ions are then detected in coincidence with photoelectrons. The molecular beam of $\mathrm{CO}_{2}, \mathrm{~N}_{2} \mathrm{O}$ and $\mathrm{C}_{2} \mathrm{H}_{2}$ crosses at right angles the VUV light beam, having the light polarization vector parallel to the synchrotron ring plane and perpendicular to the time-of-flight direction of detected ions. Incident photon fluxes and the gas pressure are monitored, and the ion yield has been corrected for flux changes of pressure and photon, when the photon energy was scanned. In order to record photoions in coincidence with photoelectrons, we used the electron-ion-ion coincidence technique. The coincidence electron-ion-ion extraction and detection system has been built following the model described in detail in Ref. 17 and its scheme can be seen in Figure 1. This device consists in a time of flight (TOF) spectrometer equipped with an ion position sensitive detector, being especially designed in order to properly measure the spatial momentum components of the dissociation ionic products [29]. The analysis of the recorded coincidences distribution (at each investigated photon energy) as a function of the arrival time differences $\left(t_{2}-t_{1}\right)$ of fragment ions to the ion position sensitive detector (shown in Figure 1), can provide, by using the procedure already applyied in several works [30-32], the following observables: (i) the kinetic energy distributions for each product ion; (ii) the life time of the intermediate molecular dications; and (iii) the angular distributions of final ions coming out from its Coulomb explosion. 


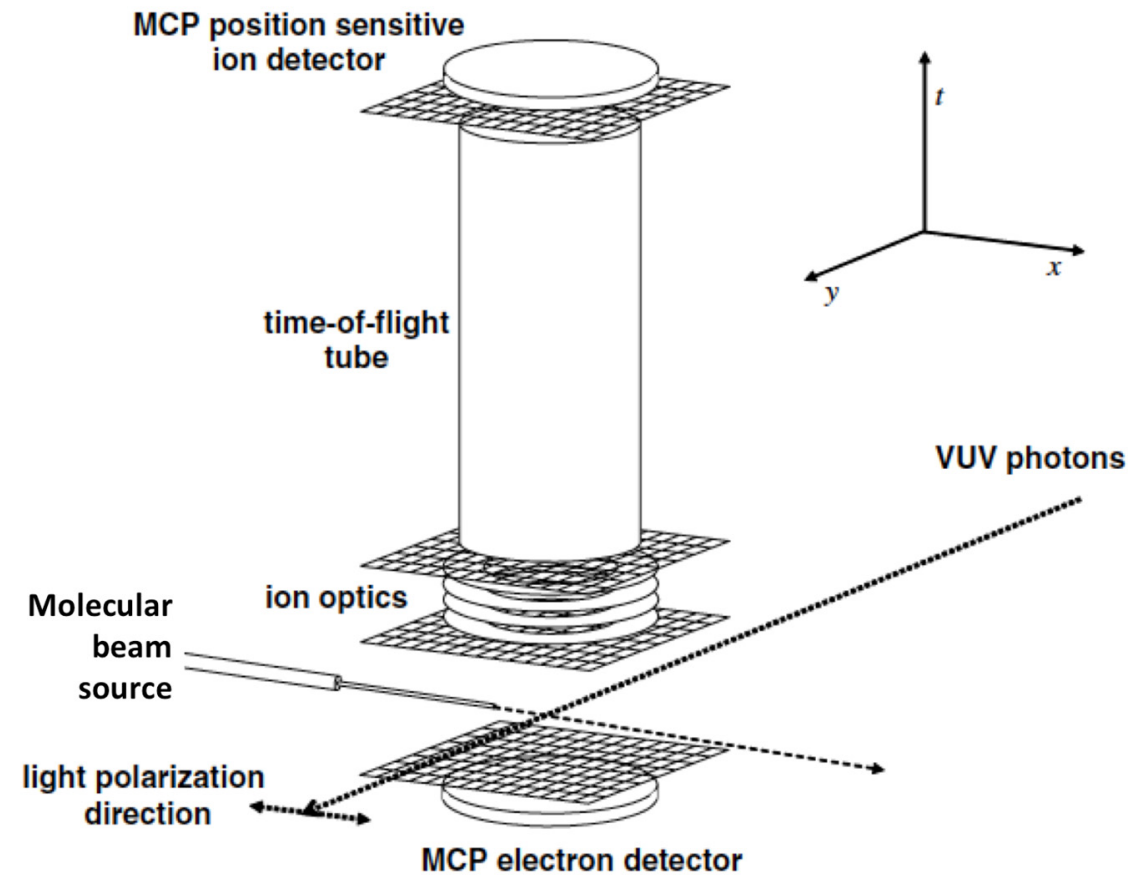

Figure 1. The scheme of the used electron-ion extraction and detection system used for the photoelectron-photoion-photoion coincidence measurements.

Carbon dioxide, nitrous oxide and acetylene, from a commercial cylinder at room temperature, were supplied to a needle effusive beam source. The used $\mathrm{CO}_{2}, \mathrm{~N}_{2} \mathrm{O}$ and $\mathrm{C}_{2} \mathrm{H}_{2}$ gases had a $99.99 \%$ and $\sim 99.0 \%$ (in the case of acetylene) nominal purity, respectively.

\section{Results and Discussion}

Carbon dioxide, nitrous oxide and acetylene are simple molecules of interest for interstellar medium ISM and planetary atmospheres (not only for the Earth but also for other planets of the Solar System like Mars, Venus and also Titan). The presence of $\mathrm{CO}_{2}, \mathrm{~N}_{2} \mathrm{O}$ in the ISM has been demonstrated by microwave spectroscopy, whereas $\mathrm{C}_{2} \mathrm{H}_{2}$ was detected by IR spectroscopic measurements [33]. Furthermore, in the Earth atmosphere carbon dioxide and nitrous oxide are important green house gases, and $\mathrm{N}_{2} \mathrm{O}$ can participate to the ozone depletion [37,38]. Acetylene was found as a minor component in the atmosphere of gas giants like the planet Jupiter, in the atmosphere of Saturn's satellite Titan [39], and in comets, where photochemical experiments have demonstrated that this simple hydrocarbon is a likely precursor of $C_{2}$, a widely observed component in such environments [34-36].

\subsection{Carbon Dioxide}

The double photoionization of $\mathrm{CO}_{2}$ was performed by using VUV synchrotron light in a photon energy range of $34-50 \mathrm{eV}$. The obtained data are in fairly good agreement with previous experiments [40-42]. By analysing recorded coincidence spectra obtained at each investigated photon energy, we were able to exctract the KER for $\mathrm{CO}^{+}$and $\mathrm{O}^{+}$fragment ions produced by the Coulomb explosion of $\mathrm{CO}_{2}{ }^{2+}$ intermediate molecular dication, formed with a threshold energy of $36.6 \mathrm{eV}$. This was possible by analyzing dimensions and shapes of the dot intensity for each recorded ion pair peak, according to the simple method suggested by Lundqvist et al. [30]. With such a procedure, we have obtained the KER distributions of each product ions for different values of the investigated photon energy (36.0, 39.0, 41.0, 44.0 and $49.0 \mathrm{eV})$ as shown in Figure 2. 


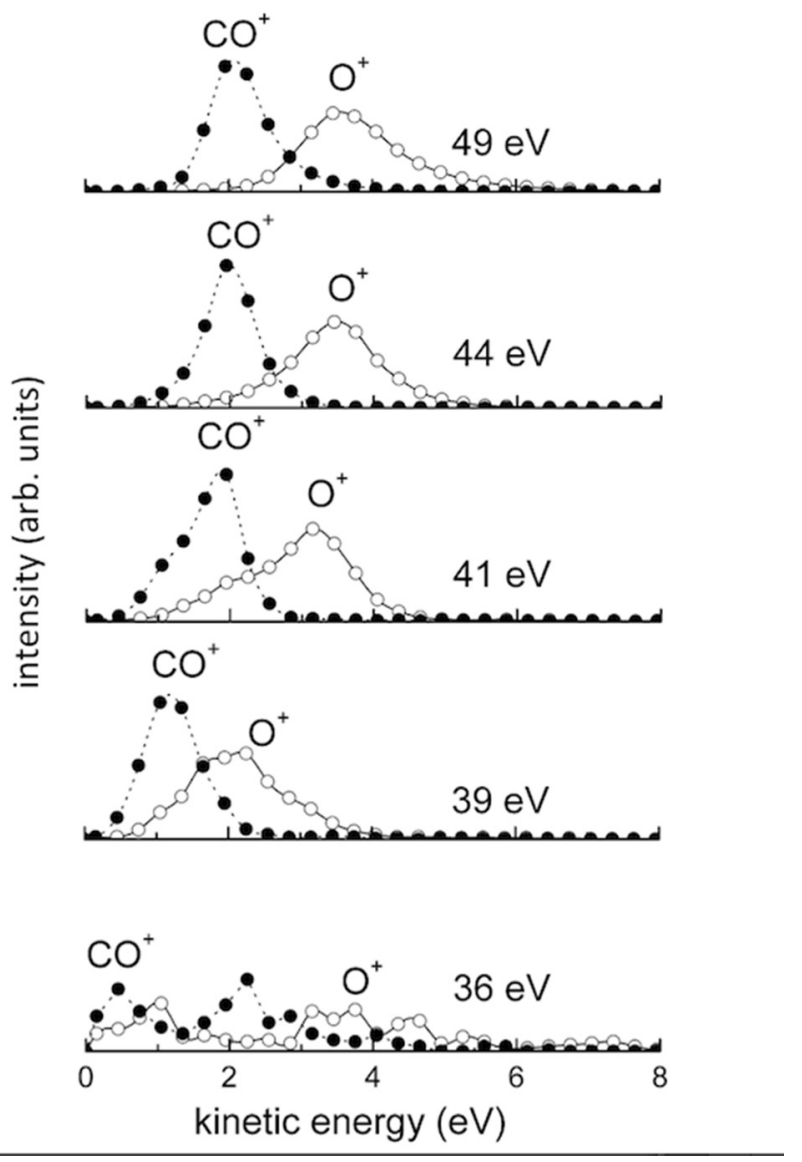

Figure 2. Kinetic energy distributions for $\mathrm{CO}^{+}$and $\mathrm{O}^{+}$fragment ions originating by Coulomb explosion of $\mathrm{CO}_{2}{ }^{2+}$ dication produced in the double VUV photoionization experiment of $\mathrm{CO}_{2}$ (see text) as a function of the photon energy.

From the kinetic energy distributions reported in Figure 2 it is evident that the KER value for the $\mathrm{O}^{+}$ions ranges between 1.0 and $5.0 \mathrm{eV}$ changing its maximum value with the investigated photon energies. This a consequence of the general behavior of doubly charged molecular ions coming from their instability towards the Coulomb explosion, with the subsequent formation of dissociative ionic fragments with a kinetic energy of several $\mathrm{eV}$, as already mentioned above. In the case of $\mathrm{O}^{+}$ formation by VUV double photoionization of carbon dioxide molecules, this energy is large enough for the atmosphere of Mars and Titan to allow these fragments to reach enough velocity to escape into space contributing to the continuous erosion of these atmospheres, as it can be appreciated by looking at the comparison with the typical escape energy of $\mathrm{O}^{+}$ions at the exobase in the atmosphere of Mars and Titan (2.0 and $0.37 \mathrm{eV}$, respectively [23]).

\subsection{Nitrous Oxide}

When nitrous oxide molecules are photoionized by using VUV synchrotron radiation in the $28-40 \mathrm{eV}$ energy range the production of $\mathrm{N}_{2} \mathrm{O}^{2+}$ molecular dications in a metatstable state is possible with a measured threshold energy of $32.2 \mathrm{eV}$, accordingly to a previous experiment [37], to which we refer for an overview of the experimental and theoretical works already performed by other laboratories. The analysis of recorded coincidence spectra at each invetigated photon energy, by using the same procedure already mentioned in the case of $\mathrm{CO}_{2}$, allow us to obtain the KER distributions of ionic fragments as a function of the investigated photon energy. They are reported in Figure 3 for the two couples of product ions $\mathrm{N}^{+}+\mathrm{NO}^{+}$and $\mathrm{O}^{+}+\mathrm{N}_{2}{ }^{+}$coming out from the Coulomb 
The 1st International Electronic Conference on Atmospheric Sciences (ECAS 2016), 16-31 July 2016;

Sciforum Electronic Conference Series, Vol. 1, 2016

explosion of the intermediate $\mathrm{N}_{2} \mathrm{O}^{2+}$ dication (the two possible two-body fragmentation processes are reported in the Figure), as a function of the analyzed photon energy: 35, 38, 41, $44 \mathrm{eV}$.
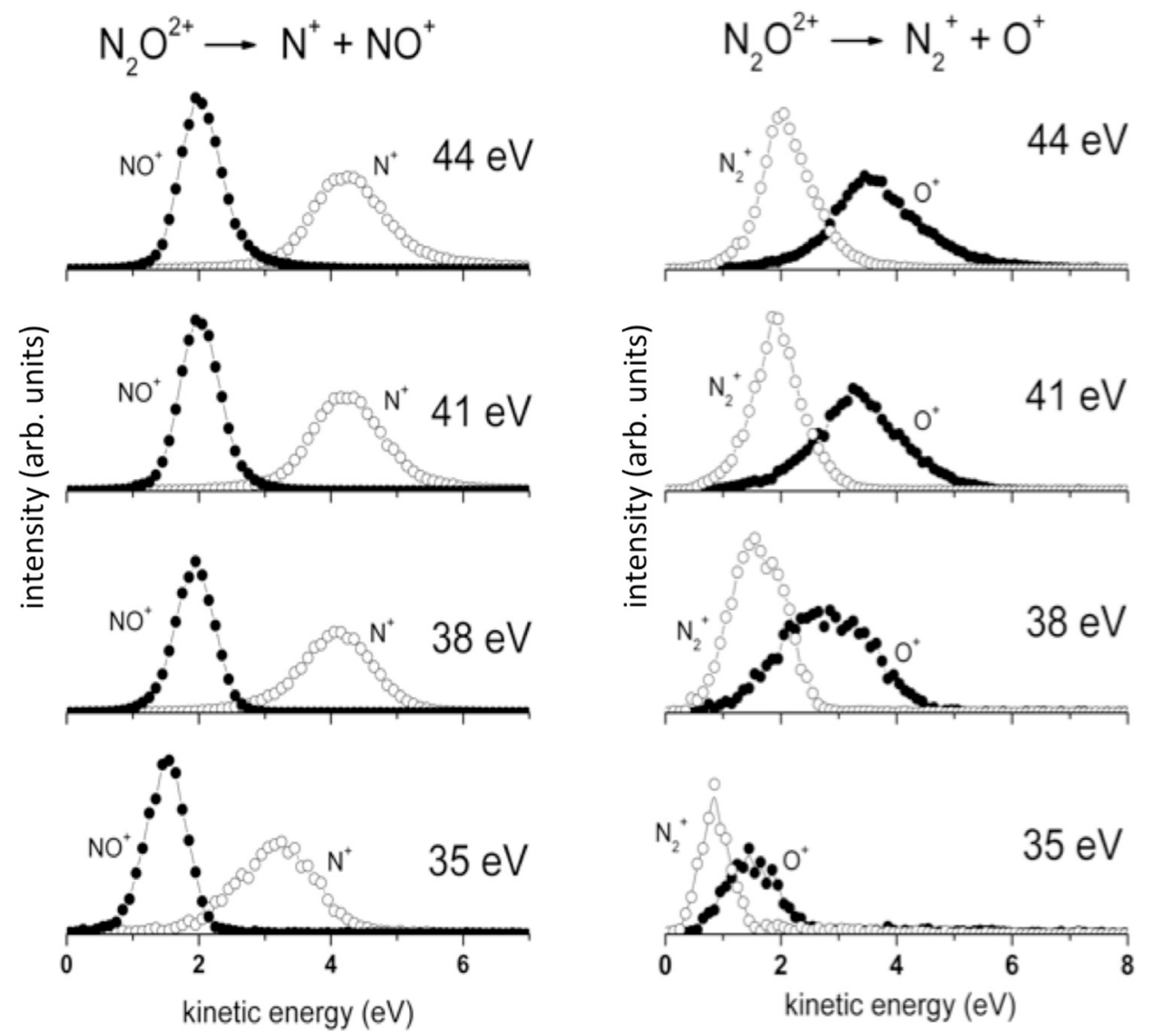

Figure 3. Kinetic energy distributions for each fragment ion $\mathrm{N}^{+}$and $\mathrm{NO}^{+}$coming from the Coulomb explosion of $\mathrm{N}_{2} \mathrm{O}^{2+}$ dication (following the two possible two-body fragmentation processes indicated on the top of the respective panel), produced in the double VUV photoionization experiment of nitrous oxide (see text), as a function of the investigated photon energy.

From Figure 3 it is possible to see that $\mathrm{N}^{+}$and $\mathrm{O}^{+}$ions $+\mathrm{N}_{2}{ }^{+}$, are formed with a KER ranging between 2.0-5.2 eV and 1.0-5.5 eV, respectively. By looking at the typical escape energy for such ions from the upper atmosphere of Mars and Titan (1.8 and $0.32 \mathrm{eV}$ (in the case of $\mathrm{N}^{+}$), 2.0 and $0.37 \mathrm{eV}$ (in the case of $\mathrm{O}^{+}$), respectively [23]), we can argue that their measured kinetic energy content is, in principle, compatible with their possible escape from the upper atmosphere of such planets.

\subsection{Ethyne}

The double photoionization of ethyne molecules by using VUV synchrotron radiation in the 32-65 eV photon energy range produces $\mathrm{C}_{2} \mathrm{H}_{2}{ }^{2+}$ dication with a measured threshold energy of 31.7 $\mathrm{eV}$. Such e dication can dissociate by Coulomb explosion via three different two-body fragmentation channels producing the following ion pairs: $\mathrm{H}^{+}+\mathrm{C}_{2} \mathrm{H}^{+}, \mathrm{CH}^{+}+\mathrm{CH}^{+}$, and $\mathrm{C}^{+}+\mathrm{CH}_{2}$. The determination of the threshold energy for such fragmentation reactions $(33.8,34.0$, and $34.0 \mathrm{eV}$, respectively) was the aim of a previous work from our laboratory [32], in agreement with experimental determinations by Eland and coworkers [43]. In the present work we have focused our attention on the KER distributions for such ions measured by using the synchrotron radiation at a photon energy of 39.0 $\mathrm{eV}$. By analyzing the density distribution of the recorded coincidence plot at such an energy, we are 
able to extract the translational energy distributions of each product ion (see Figure 4) by using the same procedure already applied to carbon dioxide and nitrous oxide double photoionization.

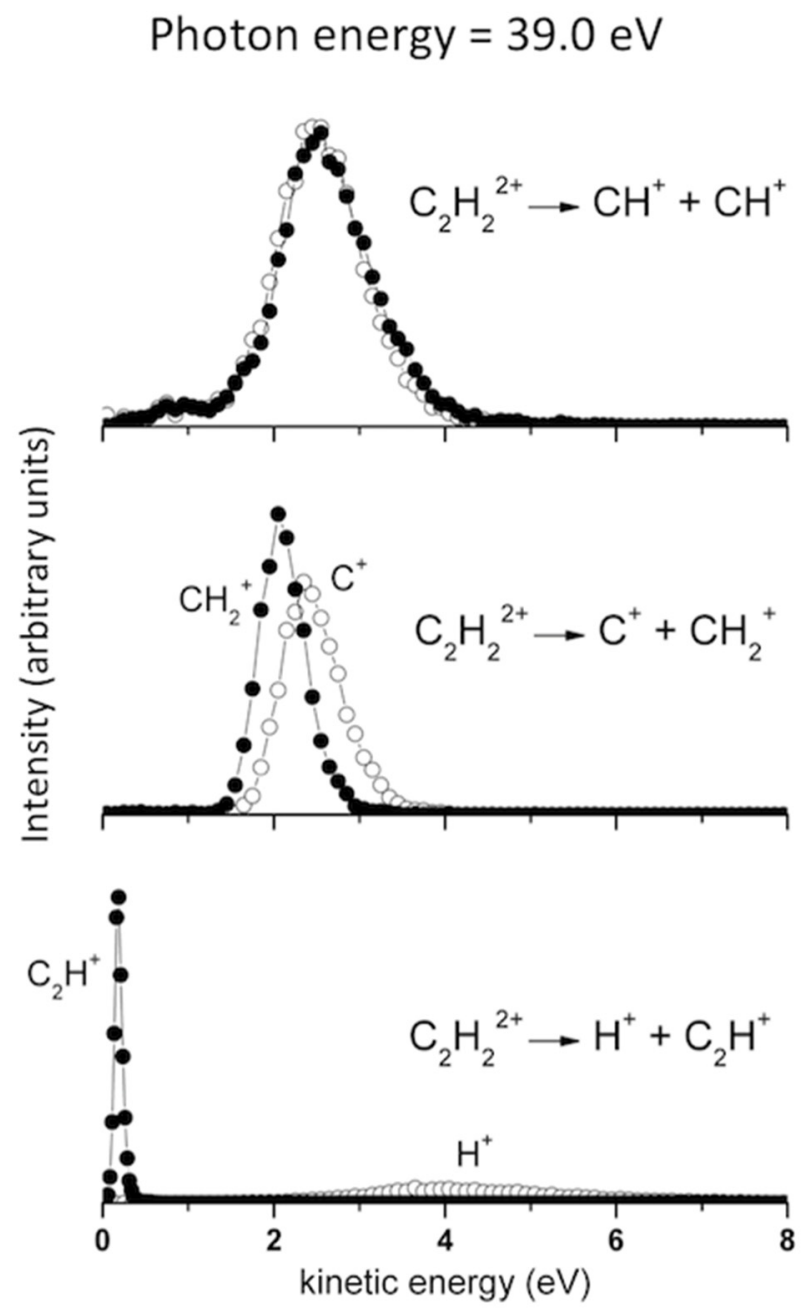

Figure 4. Kinetic energy distributions for each two-body fragment ion coming out from the Coulomb explosion of the $\mathrm{C}_{2} \mathrm{H}_{2}{ }^{2+}$ molecular dication (see reactions text), as measured at a photon energy of $39 \mathrm{eV}$.

It has to be noted that the translational energy content of each $\mathrm{H}^{+}, \mathrm{C}^{+}, \mathrm{CH}^{+}$and $\mathrm{CH}_{2}{ }^{+}$product ion is quite big, having an average value ranging between 4.0, 2.5, 2.0 and $2.5 \mathrm{eV}$, respectively. This kinetic energy is sufficient to allow this species participating in the atmospheric escape from Mars and Titan where they are characterized by a typical escape energy of 0.13 and $0.02 \mathrm{eV}$ (in the case of $\mathrm{H}^{+}$), 1.5 and $0.28 \mathrm{eV}$ (in the case of $\mathrm{C}^{+}$), 1.6 and $0.30 \mathrm{eV}\left(\right.$ for $\mathrm{CH}^{+}$), 1.8 and $0,32 \mathrm{eV}\left(\right.$ for $\mathrm{CH}_{2}^{+}$), respectively [23].

\section{Conclusions}

The dissociative double photoionization processes, induced by VUV light's photons and producing fragment ions with a high kinetic energy content, could be in general an important way for ionic species to escape from the atmosphere of some planets of the Solar System, like Mars and Titan. In fact, these processes occur via formation of intermediate molecular dications that could dissociate by Coulomb explosion towards the formation of two ionic fragment species having a kinetic energy released of several $\mathrm{eV}$, and therefore much larger than the limiting thermal escape 
The 1st International Electronic Conference on Atmospheric Sciences (ECAS 2016), 16-31 July 2016;

Sciforum Electronic Conference Series, Vol. 1, 2016

velocity characterizing some planetary atmospheres. In the case of the double VUV photoionizazion of $\mathrm{CO}_{2}, \mathrm{~N}_{2} \mathrm{O}$ and $\mathrm{C}_{2} \mathrm{H}_{2}$ molecules (in the photon energy range of $28-65 \mathrm{eV}$ ), the fragment product ions $\mathrm{O}^{+}, \mathrm{CO}^{+}, \mathrm{N}^{+}, \mathrm{CH}_{2}+\mathrm{CH}^{+}, \mathrm{C}^{+}, \mathrm{H}^{+}$are characterized by a translational energy ranging between 1.0 and $6.0 \mathrm{eV}$ that is large enough to allow their escape process from the upper atmospheres of Mars and Titan. The studies here presented could be helpful in understanding important details about the chemistry of planetary ionospheres.

Acknowledgments: Financial contributions from the MIUR (Ministero dell'Istruzione, dell'Università e della Ricerca) through PRIN 2009 (Grant 2009W2W4YF_002) project is gratefully acknowledged. The authors also gratefully thank “Fondazione Cassa di Risparmio di Perugia” for partial supports (Project code: 2014.0255.021).

Author Contributions: S.F. and S.S. conceived and designed the experiments; all authors performed the experiments; S.F., F.P., M.A., and F.V. analyzed the data; S.F., M.A., L.S., R.R., and S.S. contributed reagents/materials/analysis tools; S.F. wrote the paper.

Conflicts of Interest: The authors declare no conflict of interest. The founding sponsors had no role in the design of the study; in the collection, analyses, or interpretation of data; in the writing of the manuscript, and in the decision to publish the results.

\section{Abbreviations}

The following abbreviations are used in this manuscript:

KER: Kinetic Energy Released

VUV: Vacuum Ultraviolet

UV: Ultraviolet

ARPES: Angle-Resolved Photoemission Spectroscopy

\section{References}

References must be numbered in order of appearance in the text (including citations in tables and legends) and listed individually at the end of the manuscript. We recommend preparing the references with a bibliography software package, such as EndNote, ReferenceManager or Zotero to avoid typing mistakes and duplicated references.

Citations and References in Supplementary files are permitted provided that they also appear in the main text and in the reference list.

In the text, reference numbers should be placed in square brackets [], and placed before the punctuation; for example [1], [1-3] or [1,3]. For embedded citations in the text with pagination, use both parentheses and brackets to indicate the reference number and page numbers; for example [5] (p. 10), or [6] (pp. 101-105).

1. Brunetti, B.; Candori, P.; Ferramosche, R.; et al. Penning Ionization of C60 Molecules. Chem. Phys. Lett. 1998, 294, 584-592. Available online: http://www.sciencedirect.com/science/article/pii/S0009261498009166 (accessed on 13 June 2016).

2. Brunetti, B.G.; Candori, P.; Cappelletti, D.; et al. Penning Ionization Electron Spectroscopy of water molecules by metastable neon atoms. Chem Phys. Lett. 2012, 539-540, 19-23. Available online: http://www.sciencedirect.com/science/article/pii/S0009261412005969 (accessed on 13 June 2016).

3. Falcinelli, S.; Bartocci, A.; Cavalli, S.; Pirani, F.; Vecchiocattivi, F. The stereo-dynamics of collisional autoionization of ammonia by helium and neon metastable excited atoms through molecular beam experiments. J. Chem. Phys. 2015, 143, 164306 1-11. Available online: http://dx.doi.org/10.1063/1.4933429 (accessed on 13 June 2016).

4. Falcinelli, S.; Bartocci, A.; Cavalli, S.; Pirani, F.; Vecchiocattivi, F. Stereo-dynamics in collisional autoionization of water, ammonia, and hydrogen sulfide with metastable rare gas atoms: competition between intermolecular halogen and hydrogen bonds. Chem. Eur. J. 2016, 22, 764-771. Available online: http://onlinelibrary.wiley.com/doi/10.1002/chem.201503692 (accessed on 13 June 2016). 
The 1st International Electronic Conference on Atmospheric Sciences (ECAS 2016), 16-31 July 2016;

Sciforum Electronic Conference Series, Vol. 1, 2016

5. Falcinelli, S.; Pirani, F.; Vecchiocattivi, F. The Possible role of Penning Ionization Processes in Planetary Atmospheres. Atmosphere 2015, 6, 299-317. Available online: http://www.mdpi.com/2073-4433/6/3/299 (accessed on 13 June 2016).

6. Alagia, M.; Balucani, N.; Candori, P.; et al. Production of ions at high energy and its role in extraterrestrial environments. Rend. Lincei Sci. Fis. Nat. 2013, 24, 53-65, DOI:10.1007/s12210-012-0215-z. Available online: http://dx.doi.org/10.1007/s12210-012-0215-z (accessed on 13 June 2016).

7. Vuitton, V.; Dutuit, O.; Smith, M.A.; Balucani, N. Chemistry of Titan's atmosphere. In Titan: Surface, Atmosphere and Magnetosphere; Mueller-Wodarg, et al. Eds.; Cambridge University Press: Cambridge, UK, 2003; Chapter 7.

8. Falcinelli, S.; Rosi, M.; Candori, P.; et al. The escape probability of some ions from Mars and Titan ionospheres. ICCSA 2014, Part. I, Lecture Notes Comput. Sci. LNCS 2014, 8579, 554-570. Available online: http://link.springer.com/chapter/10.1007\%2F978-3-319-09144-0_38 (accessed on 13 June 2016).

9. Larsson, M.; Geppert, W.D.; Nyman, G. Ion chemistry in space. Rep. Prog. Phys. 2012, 75, 066901. Available online: http://www.ncbi.nlm.nih.gov/pubmed/22790651 (accessed on 14 June 2016).

10. Falcinelli, S. Penning ionization of simple molecules and their possible role in planetary atmospheres. In Recent Advances in Energy, Environment and Financial Planning-Mathematics and Computers in Science and Engineering Series; Batzias, F. et al. Eds.; WSEAS press: Athens, Greece, 2014; Volume 35, pp. 84-92.

11. Pei, L.; Carrascosa, E.; Yang, N.; Falcinelli, S.; Farrar, J.M. Velocity Map Imaging Study of Charge-Transfer and Proton-Transfer Reactions of $\mathrm{CH}_{3}$ Radicals with $\mathrm{H}_{3}{ }^{+}$. J. Phys. Chem. Lett. 2015, 6, 1684-1689. Available online: http://pubs.acs.org/doi/abs/10.1021/acs.jpclett.5b00517 (accessed on 14 June 2016).

12. Candori, P.; Falcinelli, S.; Pirani, F.; Tarantelli, F.; Vecchiocattivi, F. Interaction components in the hydrogen halide dications. Chem. Phys. Lett. 2007, 436, 322-326. Available online: http://www.sciencedirect.com/science/article/pii/S0009261407001212 (accessed on 12 June 2016).

13. Falcinelli, S.; Fernandez-Alonso, F.; Kalogerakis, K.; Zare, R.N. Mass Spectrometric Detection of Alkaline Earth Monohalide Dications. Mol. Phys. 1996, 88, 663-672. Available online: http://www.tandfonline.com/doi/abs/10.1080/00268979650026208 (accessed on 14 June 2016).

14. Tosi, P.; Correale, R.; Lu, W.; Falcinelli, S.; Bassi, D. Production of the Molecular Dication ArN ${ }^{2+}$ in the Reaction $\mathrm{Ar}^{2+}+\mathrm{N}_{2}$. Phys. Rev. Lett. 1999, 82, 450-452. Available online: http://dx.doi.org/10.1103/ PhysRevLett.82.450 (accessed on 12 June 2016).

15. Teixidor, M.M.; Pirani, F.; Candori, P.; et al. Predicted Structure and Energetics of $\mathrm{HCl}^{2+}$. Chem. Phys. Lett. 2003, 379, 139-146. Available online: http://www.sciencedirect.com/science/article/pii/S0009261403013484 (accessed on 15 June 2016).

16. Alagia, M; Brunetti, B.G., Candori, P. et al. Threshold-photoelectron-spectroscopy-coincidence study of the double photoionization of HBr. J. Chem. Phys. 2004, 120, 6985-6991. Available online: http://dx.doi.org/10.1063/1.1669382 (accessed on 15 June 2016).

17. Alagia, M.; Biondini, F.; Brunetti, B.G.; Candori, P.; et al. The double photoionization of $\mathrm{HCl}$ : An ion-electron coincidence study. J. Chem. Phys. 2004, 121, 10508-10512. Available online: http://dx.doi.org/10.1063/1.1809116 (accessed on 15 June 2016).

18. Alagia, M.; Candori, P.; Falcinelli, S.; Pirani F.; et al. Dissociative double photoionization of benzene molecules in the 26-33 eV energy range. Phys. Chem. Chem. Phys. 2011, 13, 8245-8250. Available online: http://pubs.rsc.org/en/content/articlelanding/2011/cp/c0cp02678f (accessed on 15 June 2016).

19. Pauling, L. The normal state of the helium molecule-ions $\mathrm{He}_{2}{ }^{+}$and $\mathrm{He}_{2}{ }^{+}$. J. Chem. Phys. 1933, 1, 56-59. Available online: http://dx.doi.org/10.1063/1.1749219 (accessed on 15 June 2016).

20. Nicolaides, C.A. Energy Generation from Volcanic Ground-States-Application to Cold He $2^{2+}$. Chem. Phys. Lett. 1989, 161, 547-553. Available online: http://www.sciencedirect.com/science/article/pii/ 0009261489870368 (accessed on 15 June 2016).

21. Stauber, P.; Doty, S.D.; van Dishoeck, E.F.; Benz, A.O.X-ray chemistry in the envelopes around young stellar objects. Astron. Astrophys. 2005, 440, 949-966. Available online: http://www.aanda.org/ articles/aa/abs/2005/36/aa2889-05/aa2889-05.html (accessed on 15 June 2016).

22. Thissen, R.; Witasse, O.; Dutuit, O.; Wedlund, C.S.; et al. Doubly-charged ions in the planetary ionospheres: A review. Phys. Chem. Chem. Phys. 2011, 13, 18264-18287. Available online: http://pubs.rsc. org/en/content/articlelanding/2011/cp/c1cp21957j (accessed on 15 June 2016). 
The 1st International Electronic Conference on Atmospheric Sciences (ECAS 2016), 16-31 July 2016;

Sciforum Electronic Conference Series, Vol. 1, 2016

23. Schio, L.; Li, C.; Monti, S.; Salén, P.; Yatsyna, V.; et al. NEXAFS and XPS studies of nitrosyl chloride. Phys. Chem. Chem. Phys. 2015, 17, 9040-9048. Available online: http://pubs.rsc.org/en/Content/ArticleLanding/ 2015/CP/c4cp05896h (accessed on 15 June 2016).

24. Alagia, M.; Bodo, E.; Decleva, P.; et al. The soft X-ray absorption spectrum of the allyl free radical. Phys. Chem. Chem. Phys. 2013, 15, 1310-1318. Available online: http://pubs.rsc.org/en/content/articlelanding/ 2013/cp/c2cp43466k (accessed on 15 June 2016).

25. Falcinelli, S.; Rosi, M.; Candori, P.; Vecchiocattivi, F.; Farrar, J.M.; et al. Kinetic energy release in molecular dications fragmentation after VUV and EUV ionization and escape from planetary atmospheres. Plan. Space Sci. 2014, 99, 149-157. Available online: http://www.sciencedirect.com/science/article/pii/ S0032063314001184 (accessed on 15 June 2016).

26. Blyth, R.R.; Delaunay, R.; Zitnik, M., et al. The high resolution Gas Phase Photoemission beamline, Elettra. J. Electron. Spectrosc. Relat. Phenom. 1999, 101-103, 959-964. Available online: http://www.sciencedirect. com/science/article/pii/S0368204898003818 (accessed on 15 June 2016).

27. Alagia, M.; Candori, P.; Falcinelli, S.; Lavollèe, M. Pirani, F.; et al. Dissociative double photoionization of $\mathrm{CO}_{2}$ molecules in the 36-49 eV energy range: Angular and energy distribution of ion products. Phys. Chem. Chem. Phys. 2010, 12, 5389-5395. Available online: http://pubs.rsc.org/en/content/articlelanding/ 2010/cp/b926960f (accessed on 15 June 2016).

28. Alagia, M.; Candori, P.; Falcinelli, S.; Lavollée, M.; Pirani, F.; et al. Anisotropy of the angular distribution of fragment ions in dissociative double photoionization of $\mathrm{N}_{2} \mathrm{O}$ molecules in the 30-50 eV energy range. J. Chem. Phys. 2007, 126, 201101 1-4. Available online: http://dx.doi.org/10.1063/1.2743616 (accessed on 15 June 2016).

29. Lavollée, M. A new detector for measuring three-dimensional momenta of charged particles in coincidence. Rev. Sci. Instrum. 1990, 70, 2968-2974. Available online: http://dx.doi.org/10.1063/1.1149855 (accessed on 15 June 2016).

30. Lundqvist, M.; Baltzer, P.; Edvardsson, D.; Karlsson, L.; Wannberg, B. Novel Time of Flight Instrument for Doppler Free Kinetic Energy Release Spectroscopy. Phys. Rev. Lett. 1995, 75, 1058-1061. Available online: http://dx.doi.org/10.1103/PhysRevLett.75.1058 (accessed on 15 June 2016).

31. Field, A.; Eland, J.H.D. Lifetimes of metastable molecular doubly charged ions. Chem.Phys.Lett. 1993, 211, 436-442. Available online: http://www.sciencedirect.com/science/article/pii/000926149387087J (accessed on 15 June 2016).

32. Alagia, M.; Callegari, C.; Candori, P.; Falcinelli, S.; Pirani, F.; et al. Angular and energy distribution of fragment ions in dissociative double photoionization of acetylene molecules at $39 \mathrm{eV}$. J. Chem. Phys. 2012, 136, 204302 1-6. Available online: http://dx.doi.org/10.1063/1.4720350 (accessed on 15 June 2016).

33. Witasse, O.; Dutuit, O.; Lilensten, J.; Thissen, R.; Zabka, J.; et al. Prediction of a $\mathrm{CO}_{2}{ }^{++}$layer in the atmosphere of Mars. Geophys. Res. Lett. 2002, 29, 1263 1-4. Available online: http://onlinelibrary.wiley.com/ doi/10.1029/2002GL014781/abstract (accessed on 15 June 2016).

34. Brooke, T.Y.; Tokunaga, A.T.; Weaver, H.A.; Crovisier, J.; Bockelee-Morvan, D.; Crisp, D. Detection of acetylene in the infrared spectrum of comet Hyakutake. Nature 1996, 383, 606-608. Available online: http://www.nature.com/nature/journal/v383/n6601/abs/383606a0.html (accessed on 15 June 2016).

35. Cernicharo, J.; Heras, A.M.; Pardo, J.R.; Tielens, A.G.G.M.; et al. Infrared Space Observatory's Discovery of $\mathrm{C}_{4} \mathrm{H}_{2}, \mathrm{C}_{6} \mathrm{H}_{2}$, and Benzene in CRL 618. Astrophys. J. 2001, 546, L123-L126. Available online: http://iopscience.iop.org/article/10.1086/318871/meta (accessed on 15 June 2016).

36. Woods, P.M.; Millar, T.J.; Zijlstra, A.A.; Herbst, E. The Synthesis of Benzene in the Proto-planetary Nebula CRL 618. Astrophys. J. 2002, 574, L167-L170. Available online: http://iopscience.iop.org/article/ 10.1086/342503/meta (accessed on 15 June 2016).

37. Alagia, M.; Candori, P.; Falcinelli, S.; Lavollée, M.; Pirani, F.; et al. Double photoionization of $\mathrm{N}_{2} \mathrm{O}$ molecules in the 28-40 eV energy range. Chem. Phys. Lett. 2006, 432, 398-402. Available online: http://www.sciencedirect.com/science/article/pii/S000926140601606X (accessed on 15 June 2016).

38. Biondini, F.; Brunetti, B.G.; Candori, P.; De Angelis, F. et al. Penning ionization of $\mathrm{N}_{2} \mathrm{O}$ molecules by $\mathrm{He}^{*}\left(2^{3,1} S\right)$ and $\mathrm{Ne}^{*}\left({ }^{3} P_{2,0}\right)$ metastable atoms: Theoretical considerations about the intermolecular interactions. J. Chem. Phys. 2005, 122, 164308 1-11. Available online: http://dx.doi.org/10.1063/1.1884605 (accessed on 15 June 2016). 
The 1st International Electronic Conference on Atmospheric Sciences (ECAS 2016), 16-31 July 2016;

Sciforum Electronic Conference Series, Vol. 1, 2016

39. Cravens, T.E.; Robertson, I.P.; Waite, J.H.Jr.; Yelle, R.V.; et al. Composition of Titan's ionosphere. Geophys. Res. Lett. 2006, 33, L07105. Available online: http://onlinelibrary.wiley.com/doi/10.1029/ 2005GL025575/abstract (accessed on 15 June 2016).

40. Franceschi, P.; Thissen, R.; Zabka, J.; Roithová, J., Herman, Z.; Dutuit, O. Internal energy effects in the reactivity of $\mathrm{CO}_{2}{ }^{2+}$ doubly charged molecular ions with $\mathrm{CO}_{2}$ and CO. Int. J. Mass. Spectrom. 2003, 228, 507516. Available online: http://www.sciencedirect.com/science/article/pii/S138738060300157X (accessed on 15 June 2016).

41. Slattery, A.E.; Field, T.A.; Ahmad, M.; Hall, R.I.; et al. Spectroscopy and metastability of $\mathrm{CO}_{2}{ }^{2+}$ molecular ions. J. Chem. Phys. 2005, 122, 084317. Available online: http://scitation.aip.org/content/aip/journal/ jcp/122/8/10.1063/1.1850895 (accessed on 15 June 2016).

42. Alagia, M.; Candori, P.; Falcinelli, S.; Lavollée, M.; Pirani, F.; et al. Double Photoionization of $\mathrm{CO}_{2}$ molecules in the 34-50 eV Energy range. J. Phys. Chem. A 2009, 113, 14755-14759. Available online: http://pubs.acs.org/doi/abs/10.1021/jp9048988 (accessed on 15 June 2016).

43. Thissen, R.; Delwiche, J.; Robbe, J.M.; Duflot, D.; Flament, J.P.; Eland, J.H.D. Dissociations of the ethyne dication $\mathrm{C}_{2} \mathrm{H}_{2}{ }^{2+}$. J. Chem. Phys. 1993, 99, 6590-6599. Available online: http://dx.doi.org/10.1063/1.465851 (accessed on 15 June 2016).

(C) 2016 by the authors; licensee MDPI, Basel, Switzerland. This article is an open access article distributed under the terms and conditions of the Creative Commons by Attribution (CC-BY) license (http://creativecommons.org/licenses/by/4.0/). 